\title{
The Relationship between Severity of Hearing Loss and Subjective Tinnitus Loudness among Patients Seen in a Specialist Tinnitus and Hyperacusis Therapy Clinic in UK
}

DOI: $10.3766 /$ jaaa.17144

\author{
Hashir Aazh* \\ Richard Salvi $\dagger$
}

\begin{abstract}
Background: Hearing loss is often associated with the phantom sound of tinnitus. However, the degree of the association between severity of hearing loss and tinnitus loudness taking into account the impact of other variables (e.g., emotional disturbances) is not fully understood. This is an important question for audiologists who are specialized in tinnitus rehabilitation as patients often ask whether the loudness of their tinnitus will increase if their hearing gets worse.
\end{abstract}

Purpose: To explore the relationship between tinnitus loudness and pure tone hearing thresholds.

Research Design: This was a retrospective cross-sectional study.

Study Sample: 445 consecutive patients who attended a Tinnitus and Hyperacusis Therapy Specialist Clinic in UK were included.

Data Collection and Analysis: The results of audiological tests and self-report questionnaires were gathered retrospectively from the records of the patients. Multiple-regression analysis was used to assess the relationship between tinnitus loudness, hearing loss and other variables.

Results: The regression model showed a significant relationship between the pure tone average (PTA) at the frequencies $0.25,0.5,1,2$, and $4 \mathrm{kHz}$ of the better ear and the tinnitus loudness as measured via visual analogue scale (VAS), $r$ (regression coefficient) $=0.022(p<0.001)$. Other variables significantly associated with tinnitus loudness were tinnitus annoyance $(r=0.49, p<0.001)$ and the effect of tinnitus on life $(r=0.09, p=0.006)$. The regression model explained $52 \%$ of the variance of tinnitus loudness.

Conclusions: Although increased tinnitus loudness was associated with worse PTA, the relationship was very weak. Tinnitus annoyance and impact of tinnitus on life were more strongly correlated with tinnitus loudness than PTA.

Key Words: hyperacusis, psychological assessment, pure-tone hearing thresholds, tinnitus loudness, uncomfortable loudness levels

Abbreviations: $\mathrm{BSA}=$ British Society of Audiology; $\mathrm{Cl}=$ confidence interval; HADS = hospital anxiety and depression scale; $\mathrm{HQ}=$ hyperacusis questionnaire; ISI = insomnia severity index; PTA = pure-tone average audiometric threshold; $r=$ regression coefficient; $\mathrm{SD}=$ standard deviation; $\mathrm{THI}=$ tinnitus handicap inventory; THTSC = tinnitus and hyperacusis therapy specialist clinic; ULL = uncomfortable loudness level; ULLmin = across-frequency average ULL for the ear with the lower ULL; VAS = visual analog scale

${ }^{*}$ Audiology Department, Royal Surrey County Hospital NHS Foundation Trust, Guildford, UK; $†$ Center for Hearing and Deafness, University at Buffalo, Buffalo, NY

Corresponding author: Hashir Aazh, Audiology Department, Tinnitus and Hyperacusis Therapy Specialist Clinic, Royal Surrey County Hospital, Egerton Road, Guildford GU2 7XX, UK; Email: hashir.aazh@nhs.net 


\section{INTRODUCTION}

$\mathrm{H}$ earing loss is often associated with the phantom sound of tinnitus and hyperacusis. Hyperacusis is defined as intolerance of certain everyday sounds that causes significant distress and impairment in social, occupational, recreational, and other day-to-day activities (Aazh et al, 2016). For patients with hyperacusis, sounds are often perceived as uncomfortably loud, unpleasant, frightening, or painful (Tyler et al, 2014). Most patients who experience tinnitus also have some form of hearing loss but not all patients with hearing loss have tinnitus (Tyler and Baker, 1983; Nicolas-Puel et al, 2002; Mazurek et al, 2010). The strong relationship between tinnitus and hearing impairment probably explains why, in the United Kingdom, $82 \%$ of tinnitus patients are referred to audiology departments (Gander et al, 2011). To complicate matters, some people with clinically normal hearing have tinnitus (Aazh et al, 2011), suggesting that hearing loss per se may not be a requirement for induction of tinnitus.

Based on the clinical experience of the first author, a common concern expressed by patients is that although they can cope with the current level of their tinnitus, one of their fears is that if their hearing worsens it may lead to an increase in tinnitus loudness that they would not be able to cope with. Audiologists typically reassure patients by explaining that there is no direct relationship between severity of hearing loss and tinnitus loudness. There are many people with clinically normal hearing who experience very loud tinnitus and others with very severe hearing loss, but no tinnitus. A compelling counter argument is people with acute hearing impairments such as an impacted wax, ear infections, acute noise exposure, or those wearing hearing protection experience an increase in tinnitus loudness. Consistent with this view is the observation that ear plugging leads to decreased loudness tolerance (Formby et al, 2003). Therefore, it is reasonable to assume that hearing loss severity is related to tinnitus loudness, an interpretation that is consistent with some central gain models of tinnitus and hyperacusis (Eggermont and Roberts, 2004; Auerbach et al, 2014; Chen et al, 2015a).

Interestingly, the subjective estimate of hearing loss is associated with tinnitus loudness (Hiller and Goebel, 2006). Hiller and Goebel (2006) reported that the odds ratio of people rating their tinnitus as louder than all external noises instead of rating their tinnitus as just audible in silence increases by a factor of 4.55 (95\% confidence interval [CI]: 3.51-5.91) for people with hearing loss. However, they did not assess whether the severity of hearing loss predicts the loudness of tinnitus. Savastano (2008) reported that mean values of tinnitus loudness measured via loudness matching was $15 \mathrm{~dB} \mathrm{HL}$ (standard deviation $[\mathrm{SD}]=14.3 \mathrm{~dB}$ ) for people with clinically normal hearing (i.e., pure-tone average [PTA] threshold at $0.5,1,2,4$, and $8 \mathrm{kHz}$ of $<20 \mathrm{~dB} \mathrm{HL}$ ) and $28 \mathrm{~dB}(\mathrm{SD}=16)$ for people with hearing impairment $(p=0.032)$. They did not report the mean hearing thresholds among patients that were categorized into hearing loss and normal-hearing groups. Mazurek et al (2010) also reported a significant correlation between mean PTA threshold at 0.5, 1, 2, 4, and $8 \mathrm{kHz}$ and tinnitus loudness as measured with loudness matching (regression coefficient $[r]=0.67, p<0.0001$ ). Gudwani et al (2013) likewise reported a significant correlation between tinnitus loudness as measured via loudness matching and average hearing thresholds at $0.5,1$, and $2 \mathrm{kHz}$. However, none of these studies examined whether the relationship between hearing loss and tinnitus loudness was associated with other variables known to influence tinnitus perception such as emotional states (Probst et al, 2016). Regression models can examine the degree of association between hearing loss and tinnitus loudness taking into account the impact of other variables (e.g., annoyance, anxiety) included in the regression analysis (Kutner et al, 2004). More inclusive regression models can identify the strength of the relationship between hearing loss and tinnitus loudness while taking into account other variables.

Given the ambiguities in the literature and the clinical significance of tinnitus, the aim of this study was to explore the relationship between tinnitus loudness and pure-tone hearing thresholds while taking into account the effect of other factors among patients with tinnitus and hyperacusis seen in a National Health Service audiology clinic.

\section{METHODS}

\section{Study Design and Patients}

This was a retrospective cross-sectional study conducted at the Tinnitus and Hyperacusis Therapy Specialist Clinic (THTSC), Royal Surrey County Hospital, Guildford, United Kingdom. The data included in this study were obtained from 445 consecutive patients who attended the THTSC from 2013 to 2016, for whom audiological and self-report questionnaires had been measured. In this study, $85 \%$ of the patients had been seen by an Ear, Nose, and Throat specialist and $100 \%$ had been seen by their general practitioner before being referred to the THTSC. The average age of the patients was 54.4 years $(\mathrm{SD}=15$ years, ages $=14$ 95 years). Forty-nine percent (216/445) of the patients were male.

Demographic data for the patients and the outcomes of their assessment were imported from records held at the audiology department. At THTSC, before any 
intervention, all patients undergo an assessment, which comprises the following:

- Taking a case history

- Ear examination using an otoscope

- Pure-tone audiometry

- Measurement of uncomfortable loudness levels (ULLs)

- A wide range of self-report questionnaires. These are described in more detail in the following paragraphs.

\section{Audiological Investigations}

- Pure-tone audiograms were measured using the procedure recommended by the British Society of Audiology (BSA, 2011a). The severity of hearing loss was categorized based on the PTA threshold at 0.25, 0.5, 1,2 , and $4 \mathrm{kHz}$ as recommended by BSA (2011a): mild (20-40 dB HL), moderate (41-70 dB HL), severe (71$95 \mathrm{~dB} \mathrm{HL}$ ), and profound (>95 dB HL).

- ULLs were measured following the BSA-recommended procedure (BSA, 2011b). Hyperacusis was considered as present when the average ULL at $0.25,0.5,1,2,4$, and $8 \mathrm{kHz}$ for the ear with the lower average ULL (across-frequency average ULL for the ear with the lower ULL [ULLmin]) was $\leq 77 \mathrm{~dB} \mathrm{HL}$ (Aazh and Moore, 2017). The criterion for severe hyperacusis was a ULL of $\leq 30 \mathrm{~dB}$ HL for at least one of the measured frequencies: $0.25,0.5,1,2,3,4,6$, and $8 \mathrm{kHz}$ for at least one ear (Aazh and Moore, 2018).

\section{Questionnaires}

Visual analog scale (VAS; Maxwell, 1978) scores ranged from 0 to 10 . The VAS score for loudness of tinnitus (hereafter tinnitus loudness) was assessed by asking the patient to rate the loudness of tinnitus during their waking hours over the last month. The patient was instructed that zero corresponds to no tinnitus being heard and ten is the loudest sound that they could imagine. The VAS score for annoyance induced by the tinnitus (hereafter tinnitus annoyance) was assessed by asking the patient to rate their subjective perception of annoyance on average during the last month (zero corresponds to no annoyance and ten is the most annoying thing imagined). The VAS score for the impact of tinnitus on their life (hereafter tinnitus life impact) was assessed by asking the patient to rate the effect of tinnitus on their life during the last month (zero corresponds to no effect and ten was the most extreme effect). VAS has shown to be a reliable and valid method of assessing tinnitus severity in patients with chronic tinnitus (Adamchic et al, 2012).

The tinnitus handicap inventory (THI; Newman et al, 1996), has 25 items, and handicap response choices are "no" ( 0 points), "sometimes" ( 2 points), and "yes" (4 points).
The overall score ranges from 0 to 100 . Scores from 0 to 16 indicate no handicap, scores from 18 to 36 indicate mild handicap, scores from 38 to 56 indicate moderate handicap, and scores from 58 to 100 indicate severe handicap (Newman et al, 1996).

The hyperacusis questionnaire (HQ; Khalfa et al, 2002) comprises 14 items and the response choices are "no" $(0$ points), "yes, a little" (1 points), "yes, quite a lot" (2 points), and "yes, a lot" (3 points). The overall score ranges from 0 to 42. Scores of 22 or more were taken as indicating the presence of hyperacusis handicap (Aazh and Moore, 2017).

Hospital anxiety and depression scale (HADS; Zigmond and Snaith, 1983) consists of 14 items each rated from zero to three according to the severity of difficulty experienced. The anxiety (HADS-A) and depression (HADS-D) subscale totals were calculated; total scores for each subscale range from 0 to 21 . Scores from 0 to 7 are classified as normal, scores from 8 to 10 are classified as borderline abnormal, and scores from 11 to 21 are classified as abnormal (Zigmond and Snaith, 1983).

Insomnia severity index (ISI; Bastien et al, 2001) comprises seven items that assess the severity of sleep difficulties and their effect on the patient's life. Each item is rated on a scale from 0 to 4 and the total score ranges from 0 to 28 . Scores from 0 to 7 indicate no clinically significant insomnia, scores from 8 to 14 indicate minimal insomnia, scores from 15 to 21 indicate moderate insomnia, and scores from 22 to 28 indicate severe insomnia (Bastien et al, 2001).

\section{Ethical Approval}

This study was approved by the South West-Cornwall and Plymouth Research Ethics Committee and the Research and Development Department at the Royal Surrey County Hospital.

\section{Data Analysis}

The data were anonymized before statistical analysis. Descriptive statistics (means and SDs) for the characteristics of the patients and scores for the self-report questionnaires were calculated.

Two regression analyses were conducted. First, a linear regression model was created to examine whether PTA of the better ear predicts the severity of tinnitus loudness. Second, a stepwise linear multiple regression model was created to predict the severity of tinnitus loudness, beginning with a full model that included all of the following variables: PTA of the better ear, PTA of the worse ear, ULLmin, tinnitus annoyance, tinnitus life effect, THI, HQ, ISI, HADS-A, HADS-D, age, and gender. Then, variables were removed to assess whether their inclusion significantly affected the goodness of fit. Only variables that significantly predicted tinnitus loudness remained in the final model. 
Table 1. Means and SDs of PTA Threshold at 0.25, 0.5, 1, 2, and $4 \mathrm{kHz}$ of the Better and Worse Ears, Average Uncomfortable Loudness levels (ULLs) at 0.25, 0.5, 1, 2, 4, and $8 \mathrm{kHz}$ for the Ear with the Lower Average ULL (ULLmin), Scores on the THI, HQ, Visual Analog Scale (VAS) of Tinnitus Loudness, Annoyance and Effect on Life, HADS, and ISI $(n=445)$

\begin{tabular}{lcc}
\hline Questionnaire & Mean & SD \\
\hline PTA of the better ear (dB HL) & 17.2 & 11.1 \\
PTA of the worse ear (dB HL) & 25 & 33.3 \\
ULLmin (dB HL) & 82 & 15 \\
VAS (Tinnitus loudness) & 6 & 2 \\
VAS (Tinnitus annoyance) & 6 & 2.5 \\
VAS (Effect of tinnitus on life) & 5.1 & 2.8 \\
THI & 44.9 & 24 \\
HQ & 17.6 & 9 \\
HADS (anxiety) & 8.8 & 4.7 \\
HADS (depression) & 6 & 4.6 \\
ISI & 12.3 & 7.2 \\
\hline
\end{tabular}

The $p$ value required for statistical significance was set at $p<0.05$. The STATA program (version 13) was used for statistical analyses.

\section{RESULTS}

\section{Characteristics of the Study Population}

The mean PTA at frequencies $0.25,0.5,1,2$, and $4 \mathrm{kHz}$ for the better and worse ears and the scores on the VAS, THI, HQ, HADS, and ISI for the study population are shown in Table 1. Based on the PTA for the better ear, $66 \%$ of patients (293/445) had no hearing loss, 29\% (129/ 445) had mild hearing loss, and 5\% (23/445) had moderate hearing loss. Based on the PTA for the worse ear, $49 \%$ of patients $(217 / 445)$ had no hearing loss, $36.5 \%$ (163/445) had mild hearing loss, $13 \%$ (58/445) had moderate hearing loss, $0.6 \%$ (3/445) had severe hearing loss, and $0.9 \%(4 / 445)$ had profound hearing loss.

\section{ULL and Hyperacusis}

Based on the values of ULLmin, 30\% of patients (134/ 445) had ULLs of $\leq 77 \mathrm{~dB}$ HL, which indicates hyperacusis. Four percent of patients (18/445) were diagnosed with severe hyperacusis as indicated by remarkably low ULLs of $\leq 30 \mathrm{~dB}$ HL for at least one of the measured frequencies in at least one ear. Based on scores for the HQ, 32\% (141/ 445) of patients experienced hyperacusis handicap.

\section{Tinnitus}

Based on scores for the THI, $12 \%$ of patients (52/445) had no tinnitus handicap, $32 \%$ (141/445) had a mild tinnitus handicap, 24\% (105/445) had a moderate tinnitus handicap, and 33\% (147/445) had a severe tinnitus handicap.

\section{Insomnia}

Based on scores for the ISI, 31\% (138/445) of patients did not have insomnia, 29.5\% (131/445) had mild insomnia, $27.5 \%$ (122/445) had moderate insomnia, and $12 \%$ (54/445) had severe insomnia.

\section{Depression and Anxiety}

For the depression subscale of the HADS, 67.5\% (301/ 445) of patients had normal scores, $14.5 \%(64 / 445)$ had borderline abnormal scores, and 18\% (80/445) had abnormal scores. For the anxiety subscale of the HADS, $41 \%(181 / 445)$ of patients had normal scores, $23 \%$ (102/445) had borderline abnormal scores, and 36\% (162/445) had abnormal scores.

\section{Relationship between Hearing Loss and Tinnitus Loudness}

The regression model showed a significant relationship ( $t=4.26 ; 1,443 \mathrm{df} ; p<0.001)$ between PTA of the better ear and 0-10 tinnitus loudness score (The slope of the linear fit was $0.036 r=0.036,95 \% \mathrm{CI}=$ 0.019-0.052), which indicates a 1-dB increase in PTA threshold is associated with a 0.036 increase in tinnitus loudness. This relationship is very weak and the linear model explains only $4 \%$ of the variance in tinnitus loudness suggesting that factors other than severity of hearing loss may contribute to self-report tinnitus loudness.

\section{Hearing Loss and Other Predicting Factors for Tinnitus Loudness}

To determine the contribution of other factors in tinnitus loudness, we performed a stepwise linear regression analysis that, in addition to PTA threshold of the better ear, included the PTA threshold of the worse ear, ULLmin, tinnitus annoyance, tinnitus life effect, ISI, THI, HQ, HADS-A, HADS-D, age, and gender in the linear regression model. Nine variables did not significantly increase the proportion of variance predicted by the regression model. These were the HADS depression score $(p=0.35)$, PTA thresholds for the worse ear $(p=0.47)$, THI score $(p=0.31)$, HADS anxiety score $(p=0.65), \mathrm{ULLmin}(p=0.79), \mathrm{HQ}$ score $(p=0.58)$,

Table 2. Variables Included in the Final Version of the Stepwise Linear Regression Model for Predicting VAS Tinnitus Loudness Together with Regression Coefficients $(r), p$ Values, and $95 \% \mathrm{Cl}$ Values $(n=445)$

\begin{tabular}{lllll}
\hline & \multicolumn{1}{c}{$r$} & $p$ Value & \multicolumn{2}{c}{$95 \% \mathrm{Cl}$} \\
\hline PTA of the better ear & 0.022 & $<0.001$ & 0.01 & 0.034 \\
VAS of tinnitus annoyance & 0.48 & $<0.001$ & 0.41 & 0.57 \\
VAS of effect of tinnitus on life & 0.09 & 0.006 & 0.028 & 0.17 \\
\hline
\end{tabular}


ISI score $(p=0.8)$, age $(p=0.08)$, and gender $(p=$ $0.05)$. The remaining three variables in the stepwise linear regression model that increased the proportion of variance accounted for by the model are shown in Table 2 . Tinnitus loudness was again significantly associated with PTA threshold of the better ear $(t=3.16, p<0.001, r=$ 0.022 ). However, tinnitus loudness was more strongly correlated with tinnitus annoyance $(t=2.77, p<$ $0.0001, r=0.49)$ and tinnitus life effect $(t=2.77, p<$ $0.006, r=0.10$ ) than PTA threshold of the better ear. In this three-factor linear regression model, a 1-dB increase in PTA threshold of the better ear increased the tinnitus loudness score 0.022 units. The scores on tinnitus annoyance and tinnitus life effect had larger effects on tinnitus loudness than PTA. An increase in 1 VAS unit of tinnitus annoyance was correlated with an increase of 0.49 VAS units of tinnitus loudness while an increase of 1 VAS unit of tinnitus life effect was associated with an increase of 0.1 VAS unit of tinnitus loudness. Together, the inclusion of these three factors in the linear regression model explained $52 \%$ of the variance in tinnitus loudness.

\section{DISCUSSION}

\section{Hearing Loss and Tinnitus Loudness}

Our regression analysis indicates that tinnitus loudness was weakly associated with increased PTA threshold in the better ear, which was statistically significant. One hypothesis for this positive relationship is that cochlear hearing loss leads to an increase in spontaneous activity in the central auditory system, one of the proposed mechanisms for tinnitus (Mulders and Robertson, 2009; Kaltenbach, 2011; Henry et al, 2014). The increase in spontaneous activity is assumed to be due to decrease in inhibition in the central auditory system caused by cochlear damage (Eggermont and Roberts, 2004; Chen et al, 2015a). Elevated spontaneous rates have been observed in the central auditory pathway after noise- and drug-induced hearing loss (Kaltenbach, 2006). The spontaneous rates start to increase one week posttrauma and continue to increase reaching a plateau after a few months (Kaltenbach, 2006; Mulders and Robertson, 2011). After noise exposure, spontaneous rates were elevated in the region of hearing loss; however, the increase in spontaneous rates was not tightly correlated with the amount of cochlear threshold shift or hair cell loss (Mulders et al, 2011). These physiological studies suggest that the magnitude of hearing loss may not accurately predict tinnitus severity, consistent with our results showing that PTA thresholds in the better ear only explained $4 \%$ of the variance in self-perceived tinnitus loudness. It is unclear to us why the PTA in the better ear is correlated with tinnitus loudness. One idea, largely speculative, is that hearing loss asymmetry en- hances tinnitus loudness, but we are unaware of any empirical studies that address this issue. Further work is needed to test this hypothesis.

The weak correlation between PTA and tinnitus severity and imperfect correspondence between cochlear threshold shifts and hair cell loss may be due to the fact that threshold measures do not accurately capture some forms of cochlear pathology that may trigger tinnitus. It has been proposed that tinnitus might arise from damage to inner hair cells and/or their afferent synapses; however, these forms of damage are not accurately reflected in the audiogram (Lobarinas et al, 2013; Kujawa and Liberman, 2015; Liberman and Kujawa, 2017). This may explain why some patients with little or no hearing loss have tinnitus (Savastano, 2008; Savastano et al, 2009).

In its most general form, increased central gain is hypothesized to reduce ULL and increase tinnitus loudness (Eggermont and Roberts, 2004; Auerbach et al, 2014; Chen et al, 2015a). However, one of the more well-developed central gain models by Zeng suggests that the relationships of central gain to tinnitus, hyperacusis, and hearing loss are complex (Zeng, 2013). In the Zeng model, tinnitus reduces the output dynamic range for loudness by increasing the noise floor (i.e., increased spontaneous activity). By contrast, hyperacusis reduces the input dynamic range by steepening the loudness growth function (nonlinear gain) and hearing loss reduces the input dynamic range. In the Zeng model, increased gain by itself (i.e., lower ULLs) does not induce tinnitus (hyperacusis in the absence of tinnitus). Moreover, increased gain alone does not lead to tinnitus (tinnitus in the absence of hyperacusis). One of the main limitations to testing this model is that most reports, including the present study, rely on loudness data only gathered at the endpoints of the loudness growth function, namely, tinnitus loudness at the low end and ULL at the high end.

\section{Neural Networks Mediating Tinnitus Annoyance and Tinnitus Loudness}

Tinnitus annoyance and loudness are complex emotional and perceptual phenomena that likely involve multiple regions of the central nervous system. Part of this complexity may have been captured in our final regression model, which included tinnitus annoyance and tinnitus life effect, in addition to hearing threshold, explained $52 \%$ of the variance in tinnitus loudness. Others have suggested that tinnitus loudness is greatly influenced by how much the patient is annoyed by their tinnitus and its emotional impact on their lives (Tyler and Baker, 1983; Tyler et al, 2015; Aazh et al, 2017). Modern brain imaging techniques have allowed researchers to monitor neural activity in regions of the brain implicated in emotional processes. Phantom sounds, such as real auditory and visual stimuli, can 
have emotional significance, which activate neural networks in the brain that impart biological significance to sensory experience (Anderson and Phelps, 2001; Vuilleumier and Schwartz, 2001; Schupp et al, 2003; Zeelenberg et al, 2006). The amygdala assigns emotional significance to sensory experience; some of these associations are learned (Goddard, 1964; Davis, 1992; LaBar et al, 1998). Some neural models assume that tinnitus arises from abnormal neural synchrony within the central nervous system. In tinnitus patients with significant distress, synchronized alpha band electroencephalogram activity was increased in emotional areas of the brain such as anterior cingulate cortex, insula, parahippocampus, and amygdala (Vanneste et al, 2010). Other imaging studies of acute, drug-induced tinnitus in animals have reported increased resting state activity in the amygdala and increased functional connectivity between the auditory cortex and amygdala (Chen et al, 2015a). Interestingly, transcranial magnetic stimulation of the auditory cortex reduced tinnitus loudness but did not reduce tinnitus distress (Claes et al, 2014), suggesting that the brain region regulating tinnitus distress are distinct from those involved in tinnitus loudness. Real and phantom sounds that induce negative emotions, such as anxiety, fear, and annoyance, are more likely to increase an individual's attention, consistent with functional imaging studies linking tinnitus distress with attentional networks (Schmidt et al, 2013; Husain and Schmidt, 2014; Chen et al, 2015b)

\section{Clinical Implications}

Tinnitus patients often ask whether the loudness of their tinnitus will increase if their hearing gets worse. Our results suggest that tinnitus will likely get louder, but not by very much. However, further longitudinal studies in the same subjects are needed to test the hypothesis that tinnitus will get louder as hearing loss increases. Because hearing loss increases with age and ototraumatic insults, patients should be advised to avoid loud sounds to preserve their hearing. However, prolonged daily use of hearing protection is not recommended because it could increase the risk that the tinnitus patient might develop hyperacusis (Formby et al, 2003; Aazh and Allott, 2016). On the other hand, hearing protection should be used when noise levels equal or exceed noise safety regulations. Often patients with tinnitus feel that they should protect their hearing to avoid worsening their tinnitus. Hence, some use hearing protection on a daily basis to prevent further hearing loss. These safety-seeking behaviors are likely to contribute to tinnitus-related anxiety (Bennett-Levy et al, 2004; McManus et al, 2012). Safety-seeking behaviors that restrict a patient's life experience likely contribute to tinnitus annoyance making the tinnitus "sound" even louder.
Although there is no cure for tinnitus, there are a wide range of rehabilitative approaches that can minimize tinnitus annoyance and its impact on the patient's life (Tyler et al, 2015; Aazh et al, 2016). Patients who are less annoyed by their tinnitus or who feel tinnitus does not negatively affect their life have lower tinnitus loudness ratings (Aazh et al, 2008; Aazh and Moore, 2016).

\section{Study Limitation}

As this was a cross-sectional study, the regression analyses conducted here do not directly indicate a causal link between tinnitus loudness and measured variables. In addition, this study was limited to the information that was gathered in day-to-day clinics. We did not include psychoacoustic measures of tinnitus loudness (Hoare et al, 2014). Future research should include psychoacoustic measures in addition to psychometric instruments.

\section{REFERENCES}

Aazh H, Allott R. (2016) Cognitive behavioural therapy in management of hyperacusis: a narrative review and clinical implementation. Aud Vest Res 25:63-74.

Aazh H, El Refaie A, Humphriss R. (2011) Gabapentin for tinnitus: a systematic review. Am J Audiol 20:151-158.

Aazh H, Lammaing K, Moore BCJ. (2017) Factors related to tinnitus and hyperacusis handicap in older people. Int $J$ Audiol 56:677-684.

Aazh H, Moore BC, Glasberg BR. (2008) Simplified form of tinnitus retraining therapy in adults: a retrospective study. BMC Ear Nose Throat Disord 8:7.

Aazh H, Moore BCJ. (2016) A comparison between tinnitus retraining therapy and a simplified version in treatment of tinnitus in adults. Aud Vest Res 25:14-23.

Aazh H, Moore, BCJ. (2017) Factors related to uncomfortable loudness levels for patients seen in a tinnitus and hyperacusis clinic. Int $J$ Audiol 56:793-800.

Aazh H, Moore BCJ. (2018) Prevalence and characteristics of patients with severe hyperacusis among patients seen in a tinnitus and hyperacusis clinic. J Am Acad Audiol 29:626-633.

Aazh H, Moore BCJ, Lammaing K, Cropley M. (2016) Tinnitus and hyperacusis therapy in a UK National Health Service audiology department: patients' evaluations of the effectiveness of treatments. Int J Audiol 55:514-522.

Adamchic I, Langguth B, Hauptmann C, Tass PA. (2012) Psychometric evaluation of visual analog scale for the assessment of chronic tinnitus. Am J Audiol 21:215-225.

Anderson AK, Phelps EA. (2001) Lesions of the human amygdala impair enhanced perception of emotionally salient events. Nature 411:305-309.

Auerbach BD, Rodrigues PV, Salvi RJ. (2014) Central gain control in tinnitus and hyperacusis. Front Neurol 5:206.

Bastien CH, Vallieres A, Morin CM. (2001) Validation of the insomnia severity index as an outcome measure for insomnia research. Sleep Med 2:297-307. 
Bennett-Levy J, Butler G, Fennell M, Hackmann A, Mueller M, Westbrook D. (2004) Oxford Guide to Behavioural Experiments in Cognitive Therapy. Oxford, UK: Oxford University Press.

BSA. (2011a) Pure-Tone Air-Conduction and Bone-Conduction Threshold Audiometry with and without Masking: Recommended Procedure. Reading, UK: British Society of Audiology.

BSA. (2011b) Recommended Procedure: Determination of Uncomfortable Loudness Levels. Reading, UK: British Society of Audiology.

Chen YC, Li X, Liu L, Wang J, Lu CQ, Yang M, Jiao Y, Zang FC, Radziwon K, Chen GD, Sun W, Krishnan Muthaiah VP, Salvi R, Teng GJ. (2015a) Tinnitus and hyperacusis involve hyperactivity and enhanced connectivity in auditory-limbic-arousal-cerebellar network. Elife 4:e06576.

Chen YC, Zhang J, Li XW, Xia W, Feng X, Qian C, Yang XY, Lu CQ, Wang J, Salvi R, Teng GJ. (2015b) Altered intra- and interregional synchronization in resting-state cerebral networks associated with chronic tinnitus. Neural Plast 2015: 475382 .

Claes L, Stamberger H, Van de Heyning P, De Ridder D, Vanneste S. (2014) Auditory cortex tACS and tRNS for tinnitus: single versus multiple sessions. Neural Plast 2014:436713.

Davis M. (1992) The role of the amygdala in fear and anxiety. Annu Rev Neurosci 15:353-375.

Eggermont JJ, Roberts LE. (2004) The neuroscience of tinnitus. Trends Neurosci 27:676-682.

Formby C, Sherlock LP, Gold SL. (2003) Adaptive plasticity of loudness induced by chronic attenuation and enhancement of the acoustic background. J Acoust Soc Am 114:55-58.

Gander PE, Hoare DJ, Collins L, Smith S, Hall DA. (2011) Tinnitus referral pathways within the National Health Service in England: a survey of their perceived effectiveness among audiology staff. BMC Health Serv Res 11:162.

Goddard GV. (1964) Amygdaloid stimulation and learning in the rat. J Comp Physiol Psychol 58:23-30.

Gudwani S, Munjal SK, Panda NK, Verma RK. (2013) Correlation of tinnitus loudness and onset duration with audiological profile indicating variation in prognosis. ISRN Otolaryngol 2013:205714.

Henry JA, Roberts LE, Caspary DM, Theodoroff SM, Salvi RJ. (2014) Underlying mechanisms of tinnitus: review and clinical implications. J Am Acad Audiol 25:5-22; quiz 126.

Hiller W, Goebel G. (2006) Factors influencing tinnitus loudness and annoyance. Arch Otolaryngol Head Neck Surg 132:1323-1330.

Hoare DJ, Edmondson-Jones M, Gander PE, Hall DA. (2014) Agreement and reliability of tinnitus loudness matching and pitch likeness rating. PLoS One 9:e114553.

Husain FT, Schmidt SA. (2014) Using resting state functional connectivity to unravel networks of tinnitus. Hear Res 307:153-162.

Kaltenbach JA. (2006) Summary of evidence pointing to a role of the dorsal cochlear nucleus in the etiology of tinnitus. Acta Otolaryngol Suppl 556:20-26.

Kaltenbach JA. (2011) Tinnitus: models and mechanisms. Hear Res 276:52-60.

Khalfa S, Dubal S, Veuillet E, Perez-Diaz F, Jouvent R, Collet L. (2002) Psychometric normalization of a hyperacusis questionnaire. ORL J Otorhinolaryngol Relat Spec 64:436-442.
Kujawa SG, Liberman MC. (2015) Synaptopathy in the noiseexposed and aging cochlea: primary neural degeneration in acquired sensorineural hearing loss. Hear Res Dec(330): 191-199.

Kutner MH, Nachtsheim C, Neter J. (2004) Applied Linear Regression Models. New York, NY: McGraw-Hill Education.

LaBar KS, Gatenby JC, Gore JC, LeDoux JE, Phelps EA. (1998) Human amygdala activation during conditioned fear acquisition and extinction: a mixed-trial fMRI study. Neuron 20: 937-945.

Liberman MC, Kujawa SG. (2017) Cochlear synaptopathy in acquired sensorineural hearing loss: manifestations and mechanisms. Hear Res Jun(349):138-147.

Lobarinas E, Salvi R, Ding D. (2013) Insensitivity of the audiogram to carboplatin induced inner hair cell loss in chinchillas. Hear Res 302:113-120.

Maxwell C. (1978) Sensitivity and accuracy of the visual analogue scale: a psycho-physical classroom experiment. Br J Clin Pharmacol 6:15-24.

Mazurek B, Olze H, Haupt H, Szczepek AJ. (2010) The more the worse: the grade of noise-induced hearing loss associates with the severity of tinnitus. Int J Environ Res Publ Health 7: 3071-3079.

McManus F, Van Doorn K, Viend J. (2012) Examining the effects of thought records and behavioural experiments in instigating belief change. J Behav Ther Exp Psychiatr 43:540-548.

Mulders WH, Ding D, Salvi R, Robertson D. (2011) Relationship between auditory thresholds, central spontaneous activity, and hair cell loss after acoustic trauma. J Comp Neurol 519: $2637-2647$.

Mulders WH, Robertson D. (2009) Hyperactivity in the auditory midbrain after acoustic trauma: dependence on cochlear activity. Neuroscience 164:733-746.

Mulders WH, Robertson D. (2011) Progressive centralization of midbrain hyperactivity after acoustic trauma. Neuroscience 192: $753-760$.

Newman CW, Jacobson GP, Spitzer JB. (1996) Development of the tinnitus handicap inventory. Arch Otolaryngol Head Neck Surg 122:143-148.

Nicolas-Puel C, Faulconbridge RL, Guitton M, Puel JL, Mondain M, Uziel A. (2002) Characteristics of tinnitus and etiology of associated hearing loss: a study of 123 patients. Int Tinnitus $J$ 8:37-44.

Probst T, Pryss R, Langguth B, Schlee W. (2016) Emotional states as mediators between tinnitus loudness and tinnitus distress in daily life: results from the "TrackYourTinnitus" application. Sci Rep 6:20382.

Savastano M. (2008) Tinnitus with or without hearing loss: are its characteristics different? Eur Arch Oto-Rhino-Laryngol 265: $1295-1300$.

Savastano M, Marioni G, de Filippis C. (2009) Tinnitus in children without hearing impairment. Int J Pediatr Otorhinolaryngol 73(1, Suppl):S13-S15.

Schmidt SA, Akrofi K, Carpenter-Thompson JR, Husain FT. (2013) Default mode, dorsal attention and auditory resting state networks exhibit differential functional connectivity in tinnitus and hearing loss. PLoS One 8:e76488. 
Schupp HT, Junghofer M, Weike AI, Hamm AO. (2003) Emotional facilitation of sensory processing in the visual cortex. Psychol Sci 14:7-13.

Tyler R, Noble W, Coelho C, Roncancio E, Jun H. (2015) Tinnitus and hyperacusis. In: Katz J, Chasin M, English K, Hood L, Tillery K, eds. Handbook of Clinical Audiology. Philadelphia, PA: Wolters Kluwer, 647-658.

Tyler RS, Baker LJ. (1983) Difficulties experienced by tinnitus sufferers. J Speech Hear Disord 48:150-154.

Tyler RS, Pienkowski M, Roncancio ER, Jun HJ, Brozoski T, Dauman N, Dauman N, Andersson G, Keiner AJ, Cacace AT, Martin N, Moore BC. (2014) A review of hyperacusis and future directions: part I. definitions and manifestations. Am J Audiol 23:402-419.
Vanneste S, Plazier M, der Loo Ev, de Heyning PV, Congedo M, De Ridder D. (2010) The neural correlates of tinnitus-related distress. Neuroimage 52:470-480.

Vuilleumier P, Schwartz S. (2001) Emotional facial expressions capture attention. Neurology 56:153-158.

Zeelenberg R, Wagenmakers EJ, Rotteveel M. (2006) The impact of emotion on perception: bias or enhanced processing? Psychol Sci 17:287-291.

Zeng FG. (2013) An active loudness model suggesting tinnitus as increased central noise and hyperacusis as increased nonlinear gain. Hear Res 295:172-179.

Zigmond AS, Snaith RP. (1983) The hospital anxiety and depression scale. Acta Psychiatr Scand 67:361-370. 